www.jmscr.igmpublication.org

Index Copernicus Value: 79.54

ISSN (e)-2347-176x ISSN (p) 2455-0450

crossrefDOI: https://dx.doi.org/10.18535/jmscr/v7i2.14

\title{
Clinicopathological Spectrum of Uterine Sarcoma
}

\author{
Authors
}

Anila Tresa Alukal ${ }^{1}$, Swathi B Shankar ${ }^{2}$

${ }^{1,2}$ Former Senior Resident, Department of Obstetrics and Gynaecology, Govt Medical College, Thrissur

*Corresponding Author

Swathi B Shankar

Email: swathibshankar.87@gmail.com

\begin{abstract}
Uterine sarcomas constitute about $1 \%$ of female genital tract malignancies. And $3 \%$ to $7 \%$ of Uterine cancer. Uterine sarcomas have an aggressive behaviour. Rarity of the tumour and histopathological diversity has contributed to the lack of consensus on risk factors. The aim of the study was to assess the epidemiological aspects, clinical features and histopathological features of uterine sarcomas.

Materials and Methods: A retrospective record based study of 32 diagnosed cases of uterine sarcoma over a period of 5 years (Jan 2010- Dec 2015) who had attended Dept. of Obstetrics and Gynaecology, Govt. Medical College Thrissur.Data was collected from the hospital records of 5 years. Variables studied include histology, age, parity, menopausal status, presenting symptoms, clinical features, co-morbidities, ultrasound findings, preoperative diagnosis, and stage of the disease.

Results: The most common histologic variety is Mixed malignant mullerian tumor (44\%) followed by Leiomyosarcoma. (22\%) MMMT is commonly seen in postmenopausal age group (79\%) Leiomyosarcoma and Endometrial stromal sarcoma is mostly seen in the perimenopausal age group (45\% \& $57 \%$ respectively).
\end{abstract}

Keywords: uterine sarcoma; clinical features; pathological spectrum.

\section{Introduction}

Uterine sarcomas are rare tumours that account for $1 \%$ of female genital tract malignancies and $3 \%$ to $7 \%$ of uterine cancers ${ }^{1}$.Although the aggressive behaviour of most cases is well recognized, their rarity and histopathological diversity has contributed to the lack of consensus on risk factors for poor outcome and optimal treatment ${ }^{2}$. Histologically, uterine sarcomas were first classified into carcinosarcomas, accounting for $40 \%$ of cases, leiomyosarcomas (40\%), endometrial stromal sarcomas (10\% to $15 \%)$, and undifferentiated sarcomas (5\% to $10 \%$ ). Recently, carcinosarcoma has been reclassified as a dedifferentiated or metaplastic form of endometrial carcinoma. Despite this, and probably because it behaves more aggressively than the ordinary endometrial carcinoma, carcinosarcoma is still included in most retrospective studies of uterine sarcomas, as well as in the 2003 World Health Organization (WHO) classification

The clinical presentation of uterine sarcomas is nonspecific and dependent of histologic subtype. Classically, they present as a rapidly growing pelvic mass, which may be accompanied by vaginal bleeding and abdominal or pelvic pain ${ }^{3}$.

Leiomyosarcoma is the most common histological subtype of uterine sarcomas. The great majority 
arise de novo, but rarely (in $0.2 \%$ of cases) it may result from a sarcomatous transformation in a benign leiomyoma ${ }^{4}$. It is characterized by an aggressive behaviour, with a five-year survival rate ranging from $18.8 \%$ to $68 \%$, which varies widely according to different stages. Low-grade and serosal involvement seem to be significant prognostic factors ${ }^{5}$. Most leiomyosarcomas occur in women over 40 years of age, with a median age of 60 years. Long-term tamoxifen use and prior pelvic radiation seem to be associated with a small increase in risk ${ }^{6}$.Signs and symptoms are similar to those occurring with leiomyomas, and include abnormal vaginal bleeding (56\%), palpable pelvic mass $(54 \%)$ and pelvic pain $(22 \%)^{7}$.

Endometrial stromal sarcoma is composed of cells that resemble endometrial stromal cells of the proliferative endometrium.It accounts for $0.2 \%$ of all malignant uterine tumours and $10 \%-15 \%$ of uterine malignancies with a mesenchymal component. It is a low-grade, well-differentiated tumour without significant cellular atypia ${ }^{8}$. ESS is a relatively indolent lesion, generally with a favourable prognosis, with five- and 10-year survival rates of $98 \%$ and $89 \%$ for stage I disease, which corresponds to the majority of patients at presentation. The outcome is largely dependent on the extent of the tumour at presentation, and stage is the most significant indicator for survival .ESS occurs more commonly in women between 40 and 55 years of age ${ }^{9}$. There has been a reported association with tamoxifen and oestrogen use. They usually present with abnormal vaginal bleeding, pelvic pain, and dysmenorrhea; however around $25 \%$ of patients are asymptomatic ${ }^{10}$.

Carcinosarcoma, also referred to as "malignant mixed mulleintumour," is a biphasic neoplasm composed of distinctive and separate, but admixed, malignant-appearing epithelial and mesenchymal elements ${ }^{11}$. It accounts for almost half of all uterine sarcomas. It is typically in postmenopausal women. Up to $37 \%$ of patients with carcinosarcomas have a history of pelvic irradiation $^{12}$. These tumours tend to occur in youngerwomen, often contain heterologous elements, and are found at advanced stage ${ }^{13}$. Carcinosarcomas are typically large, bulky polypoid masses, fillingthe uterine cavity and prolapsing through the cervical os. The cut surface is fleshy and shows areas of haemorrhage, necrosis, and cystic change. Myometrial invasion is also seen ${ }^{14}$.

\section{Aim of the Study}

To assess the epidemiological aspects, clinical features and histopathological features of uterine sarcomas.

\section{Materials and Method}

A retrospective record based study of 32 diagnosed cases of uterine sarcoma over a period of 5 years (Jan 2010- Dec 2015) who had attended Dept. of Obstetrics and Gynaecology, Govt. Medical College Thrissur.Data was collected from the hospital records of 5 years. Variables studied include histology, age, parity, menopausal status, presenting symptoms, clinical features, comorbidities, ultrasound findings, preoperative diagnosis, stage of the disease

\section{Statistical Analysis}

Data analysis - Epi Info software. Qualitative variables were assessed as proportions. Relations between variables was tested by Chi square test.

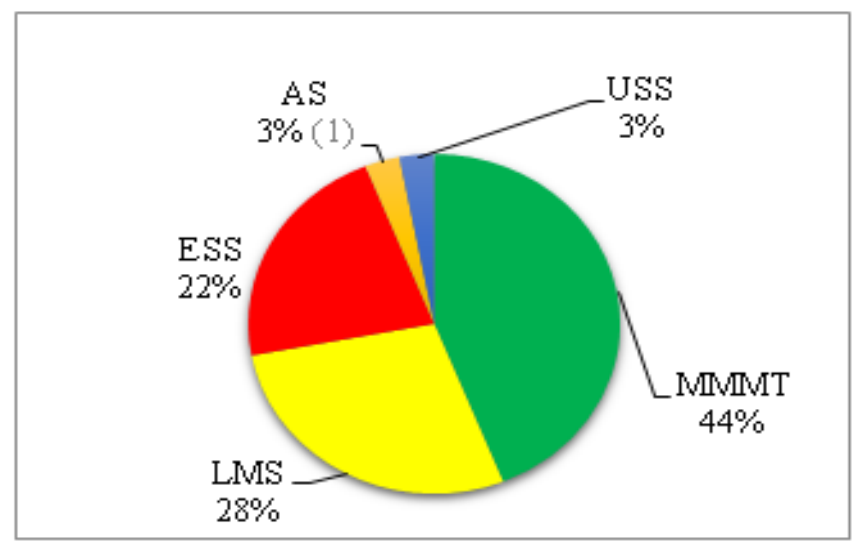

Fig 1- Frequency of Uterine Sarcoma

\section{Observations}

The most common histological pattern was carcinosarcoma or Mixed malignant mullerian tumour.ie $44 \%$ of the cases, followed by 
leiomyosarcoma 28\%, endometrial stromal sarcoma $22 \%$ and undifferentiated stromal sarcoma and adenosarcoma 3\% each.

The most common age group in which uterine sarcoma was seen is $40-49$ i.e. $50 \%$. Followed by $22 \%$ in 50 - 59 years of age and $20 \%$ in above 60 years and $6 \%$ in $30-39$ yrs. of age.

Majority of the patients were multiparous $-67 \%$. Also, majority of the patients were obese $-61 \%$. Uterine sarcomas were seen mostly in postmenopausal age group - 50\% followed by perimenopausal age group $-40 \%$.

MMMT was commonly seen in 60 and above age group (postmenopausal group).Whereas leimyosarcoma and endometrial sarcoma were seen in 40-49 yrs. of age (perimenopausal group) The most common presenting complaint of MMMT was postmenopausal bleeding. (50\%) followed by discharge per vagina and abdominal pain. The most common presenting complaint for leiomyosarcoma and endometrial stromal sarcoma was heavy menstrual bleeding (67 \% and 43\% respectively) followed by abdominal pain.

About $53 \%$ patients had severe anaemia on presentation. Only $13 \%$ patients had a family history of malignancy. There was no prior history of radiation exposure, tamoixifen therapy or oestrogen replacement therapy in the study group. MMMT most commonly presented in Stage Ib (79\%), leimyosarcoma in stage IIa (40\%) and endometrial stromal sarcoma in stage IB $(57 \%)$

\section{References}

1. Bonder-Adler B, Bodner K, Obermair A, et al (2001). Prognostic parameters in carcinosar-comas of the uterus: a clinicopathologic study. Anticancer Res, 21, 3069-74.

2. Brooks SE, Zhan M, Cote T, Baquet CR (2004). Surveillance, epidemiology, and end results analysis of 2677 cases of uterine sarcoma 1989-1999. Gynecol Oncol, 93, 204-8.

3. Fujita H, Adachi S, Kigawa J, et al (2004). A clinicopathological study of uterine sarcoma in last decade - a retrospective study of KCOG/USSG inter group study. Adv Obstet Gynecol, 56,

4. Giuntoli II RL, Metzinger DS, Dimarco CS et al(2003). Retrospective rewiew of 208 patients with lyomyosarcoma of the uterus: prognostic indicators, surgical management, and adjuvant therapy. Gynecol Oncol, 89, 460-9.

5. D'Angelo E, Prat J. Uterine sarcomas: a review. Gynecol Oncol 2010; 116: 131-139.

6. Acharya S, Hensley ML, Montag AC, Fleming GF. Rare uterine cancers. Lancet Oncol 2005; 6: 961-971.

7. Hubalek M, Ramoni A, Mueller-Holzner E, Marth C. Malignant mixed mesodermal tumor after tamoxifen therapy for breast cancer. Gynecol Oncol 2004; 95: 264-266.

8. Wu TI, Yen TC, Lai CH. Clinical presentation and diagnosis of uterine sarcoma, including imaging. Best Pract Res Clin Obstet Gynaecol 2011; 25:681-689.

9. Shaan SH, Jagannathan JP, Krajewski K, O'Regen KN, George S, Ramaiya NH. Uterine sarcomas: then and now. AJR Am J Roentgenol 2012; 199:213-223.

10. Memarzadeh S, Mundt AJ, Berek JS. Uterine sarcoma: Classification, clinical manifestations, and diagnosis. In: UpToDate, Goff B, Falk SJ, eds. 2012.

11. Seddon BM, Davda R. Uterine sarcomas recent progress and future challenges. Eur $\mathbf{J}$ Radiol 2011; 78:30-40.

12. Tse KY, Crawford R, Ngan HYS. Staging of uterine sarcomas. Best Pract Res Clin Obstet Gynaecol 2011; 25:733-749.

13. D'Angelo A, Prat J. Uterine sarcomas: a review. Gynecol Oncol 2010; 116:131-139.

14. Amant F, Coosemans A, Debiec-Rychter M, Timmerman D, Vergote I. Clinical management of uterine sarcomas. Lancet Oncol 2009; 10:1188-11981 